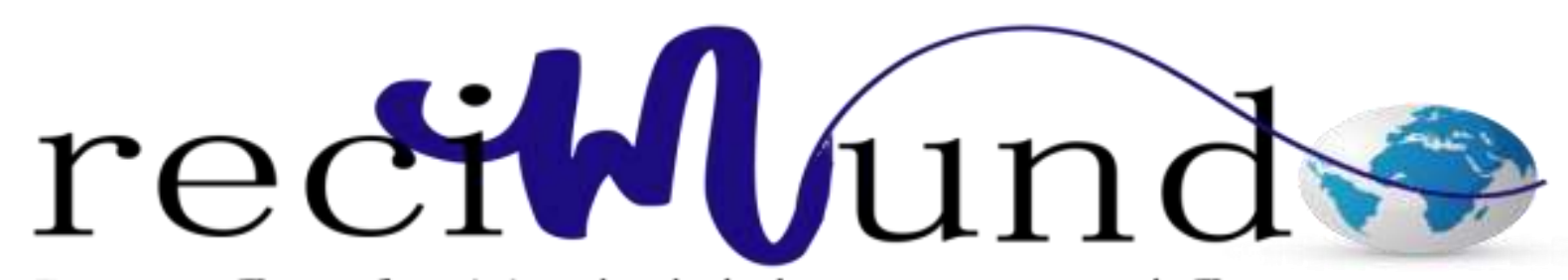

Revista Científica Mundo de la Investigación y el Conocimiento

José Luis Maldonado López a; Ivonne Carolina Recalde Tello ${ }^{\text {b; }}$ Angela Mishel

Torres Cadena ${ }^{\text {c; }}$ Winna Viviana Arévalo Vaca ${ }^{\mathrm{d}}$

Manejo de la diabetes gestacional

Management of gestational diabetes

Revista Científica Mundo de la Investigación y el Conocimiento. Vol. 3 núm.3. Esp., noviembre, ISSN: 2588-073X, 2019, pp. 297-315

DOI: $10.26820 /$ recimundo/3.(3.Esp).noviembre.2019.297-315

URL: http://recimundo.com/index.php/es/article/view/607

Código UNESCO: 3205 Medicina Interna

Tipo de Investigación: Artículo de Revisión

(C) RECIMUNDO; Editorial Saberes del Conocimiento, 2019

Recibido: 15/09/2019

Aceptado: 23/10/2019

Publicado: 30/11/2019

Correspondencia: jlmaldonadol@ hotmail.com

\footnotetext{
a. Médico Cirujano; Investigador Independiente; Guayaquil, Ecuador; jlmaldonadol@ @otmail.com

b. Médica Cirujana; Investigadora Independiente; Guayaquil, Ecuador.

c. Médica Cirujana; Investigadora Independiente; Guayaquil, Ecuador; mishut@ outlook.com

d. Médica; Investigadora Independiente; Guayaquil, Ecuador; warevaloczs5@ gmail.com
} 


\section{Manejo de la diabetes gestacional}

Vol. 3, núm. 3 Esp., (2019)

José Luis Maldonado López; Ivonne Carolina Recalde Tello; Angela Mishel Torres Cadena; Winna Viviana Arévalo Vaca

\section{RESUMEN}

La obesidad lleva a condiciones de alto riesgo durante el embarazo, como ser aborto espontáneo, diabetes gestacional, hipertensión gestacional, preeclampsia, parto prematuro de Indicación médica, alteraciones del trabajo de parto y mayor tasa de cesárea incluso ciertos tipos de cáncer. En este caso se estudia la condición de diabetes gestacional en mujeres embarazadas y el riesgo que tiene tanto para la mujer como para el feto y posterior bebe. La metodología utilizada en la investigación es de tipo bibliográfica. Las conclusiones generales a las que se pueden llegar es que los estilos de vida saludable y dietas sanas contribuyen a reducir los riesgos de padecer una diabetes gestacional durante el embarazo. En los casos donde se ha diagnosticado diabetes gestacional, la recomendación aparte de una buena nutrición es la utilización de la metformina inclusive más que la insulina que es el último recurso empleado y esto debido a que cuando se hace la comparación entre ambos medicamentos, la metformina es menos invasiva y tiene mayores éxitos en las llegadas a término de los embarazos.

Palabras Clave: Metformina; Gestacional; Dieta; Embarazo; Glicemia. 


\title{
Manejo de la diabetes gestacional
}

Vol. 3, núm. 3 Esp., (2019)

José Luis Maldonado López; Ivonne Carolina Recalde Tello; Angela Mishel Torres Cadena;

Winna Viviana Arévalo Vaca

\begin{abstract}
Obesity leads to high risk conditions during pregnancy, such as spontaneous abortion, gestational diabetes, gestational hypertension, preeclampsia, premature delivery of medical indication, alterations in labor and a higher caesarean section, including certain types of cancer. In this case, the condition of gestational diabetes in pregnant women and the risk it has for both the woman and the fetus and subsequent baby are studied. The methodology used in the research is bibliographic. The general conclusions that can be reached is that healthy lifestyles and healthy diets contribute to reducing the risks of suffering from gestational diabetes during pregnancy. In cases where gestational diabetes has been diagnosed, the recommendation apart from good nutrition is the use of metformin even more than the insulin that is the last resources used and this is because when the comparison between both drugs is made, metformin It is less invasive and has greater successes in term pregnancies.
\end{abstract}

Keywords: Metformin; Gestational; Diet; Pregnancy; Glycemia. 


\section{Manejo de la diabetes gestacional}

Vol. 3, núm. 3 Esp., (2019)

José Luis Maldonado López; Ivonne Carolina Recalde Tello; Angela Mishel Torres Cadena; Winna Viviana Arévalo Vaca

\section{Introducción.}

La diabetes gestacional (DMG) es la tercera gran categoría clínica en la clasificación actual de la diabetes, y representa un factor de riesgo para la mujer y un problema de salud para el producto. A pesar de las limitaciones persistentes para detectarla, la DMG se identificó hace muchos años: la primera descripción reconocida de la DMG apareció en 1882, aunque el primer caso se había descrito en 1824 y se define como cualquier grado de intolerancia a los hidratos de carbono que se inicia o se detecta por primera vez durante el embarazo. Si no se controla, la DMG representa un problema de salud, de alto costo y de graves consecuencias para el feto y para la mujer. La Asociación Americana de Diabetes calcula que la DMG ocurre en aproximadamente $7 \%$ de todos los embarazos en Estados Unidos, lo que equivale a más de 200,000 casos anuales (Duarte-Gardea, Muñoz, Rodríguez-Saldaña, \& Escorza-Domínguez, 2004).

La diabetes gestacional incrementa el riesgo de anomalías esqueléticas como el síndrome de regresión caudal, anomalías espinales y siringomielia; a nivel renal hidronefrosis, agenesia renal y quistes renales. Las malformaciones intestinales más comunes son: atresia del duodeno y el recto o en cualquier parte del tracto gastrointestinal. El diagnóstico se realiza por medio de la prueba de tolerancia a la glucosa, la cual no debe ser mayor de $140 \mathrm{mg} / \mathrm{dl}$, esta prueba tiene falsos negativos hasta en un $10 \%$ de los casos debido a emesis durante la prueba. La diabetes gestacional altera diversos sistemas en el feto, el pobre control glicémico desde el inicio del embarazo afecta la organogénesis y el control tardío la composición corporal, incluyendo macrosomía y dificultad respiratoria (Arizmendi, Pertuz, Colmenares, Hoyos, \& Palomo, 2012, pág. 51). 


\section{Manejo de la diabetes gestacional}

Vol. 3, núm. 3 Esp., (2019)

José Luis Maldonado López; Ivonne Carolina Recalde Tello; Angela Mishel Torres Cadena;

Winna Viviana Arévalo Vaca

La diabetes preexistente o pregestacional expone al feto a concentraciones elevadas de glucosa durante el primer trimestre del embarazo, incrementando el riesgo de aborto espontáneo y malformaciones congénitas (especialmente del sistema nervioso central, cardiovascular, renal y musculoesquelético). La probabilidad de malformaciones y aborto tiene una relación lineal con la concentración de glucosa plasmática de la madre, expresada en la concentración de hemoglobina glucosilada (HbA1c); el riesgo excesivo se puede reducir cuando la madre mantiene un excelente control glucémico en el primer trimestre del embarazo (Font-López, y otros, 2010, págs. 673-674).

Aún es discutible si la prueba de detección de DG deba realizarse de manera universal o selectiva, ni siquiera se ha establecido en qué semana de la gestación es el tiempo óptimo. Tomando en cuenta que la etapa de mayor aparición ocurre después de la segunda mitad del embarazo, lo más común es que la prueba se realice entre las semanas 24 y 28 de gestación. Aunque se han propuesto diferentes esquemas para la prueba de detección, el más común consiste en la administración por vía oral de 50 g de glucosa, en cualquier hora del día, y obtener una muestra de sangre una hora después para medir la glucosa plasmática. Se descarta DG si las cifras de glucemia no exceden los límites entre 130 y 140 mg/dL (Zárate, Hernández-Valencia, \& Saucedo, La detección y manejo de la diabetes gestacional. Controversias, críticas y comentarios, 2008, pág. 131).

La obesidad lleva a condiciones de alto riesgo durante el embarazo, como ser aborto espontáneo, diabetes gestacional, hipertensión gestacional, preeclampsia, parto prematuro de Indicación médica, alteraciones del trabajo de parto y mayor tasa de cesárea incluso ciertos tipos de cáncer. La obesidad en el embarazo es un conflicto para salud pública, pues incrementa 


\section{Manejo de la diabetes gestacional}

Vol. 3, núm. 3 Esp., (2019)

José Luis Maldonado López; Ivonne Carolina Recalde Tello; Angela Mishel Torres Cadena; Winna Viviana Arévalo Vaca

riesgos obstétricos y neonatales aumenta el riesgo de presentar enfermedades y complicaciones durante el embarazo y el parto como: Diabetes gestacional, preclampsia, enfermedades hepáticas no alcohólicas, trastornos de la coagulación (tromboembolias) y oligo/polihidramnios y en el feto los trastornos abarcan: macrosomía fetal, síndrome de distress respiratorio y productos con bajo peso para la edad gestacional, prematurez, malformaciones genéticas y aumento de riesgo de muerte fetal. La obesidad se clasifica según la OMS en: Normo peso (IMC de 18.5-24.9), exceso de peso (IMC>25 kg/m2), sobrepeso o pre obeso (IMC 25-29.9 kg/m2), obesidad grado I o moderada (IMC 30-34.9 kg/m2), obesidad grado II severa (IMC de 35-39.9 kg/m2), obesidad grado III o mórbida (IMC>40kg/m2) (Bustillo, y otros, 2016, pág. 2).

Antes del descubrimiento de la insulina, el tratamiento era principalmente dietético, empleándose aceite de oliva y otras grasas para suplir las calorías derivadas del uso de los carbohidratos y limitar así las hiperglucemias. Luego de la obtención de esta hormona por Bantig y Best en 1922 y de su posterior aplicación clínica, se produjo un acontecimiento revolucionario en el manejo de las pacientes diabéticas embarazadas y se redujo de forma dramática la morbimortalidad materno-fetal (Rimbao Torres, y otros, 2007).

Imagen 1. Incremento de peso respecto al índice de masa corporal al inicio del embarazo

\begin{tabular}{|cc|}
\hline IMC & $\mathrm{Kg}$ \\
& \\
$<18$ & $13-18$ \\
$<25$ & $11-16$ \\
$25-30$ & $7-11$ \\
$>30$ & $5-9$ \\
\hline
\end{tabular}




\section{Manejo de la diabetes gestacional}

Vol. 3, núm. 3 Esp., (2019)

José Luis Maldonado López; Ivonne Carolina Recalde Tello; Angela Mishel Torres Cadena;

Winna Viviana Arévalo Vaca

Fuente: (Zárate, Hernández-Valencia, \& Saucedo, La detección y manejo de la diabetes gestacional. Controversias, críticas y comentarios, 2008).

Imagen 2. Mujeres con análisis de glucemia antes y después del parto

\begin{tabular}{|ccc|}
\hline Edad & Durante el embarazo & En el posparto \\
& $\%$ & $\%$ \\
$18-24$ & - & 12 \\
$25-29$ & 73 & 17 \\
$30-34$ & 77 & 17 \\
$35-40$ & 81 & 25 \\
\hline
\end{tabular}

Fuente: (Zárate, Saucedo, Basurto, \& Hernández, 2011).

De manera general se va aceptando que la diabetes gestacional no es una forma diferente de diabetes sino una etapa de la misma enfermedad que ocurre en personas predispuestas genéticamente en la que se suma el impacto del estilo de vida. El aumento en la prevalencia de obesidad y el retardo en la edad de inicio de la etapa reproductiva de la mujer sustentan la teoría de un aumento en la ocurrencia de la diabetes gestacional (Zárate, Saucedo, Basurto, \& Hernández, 2011, pág. 2).

\section{Metodología.}

La metodología de la investigación es de tipo documental y bibliográfica en vista de que se recurrió a medios electrónicos para obtener la información que da soporte a este trabajo, se consultaron varios trabajos realizados por otros autores donde exponen de manera clara y 


\section{Manejo de la diabetes gestacional}

Vol. 3, núm. 3 Esp., (2019)

José Luis Maldonado López; Ivonne Carolina Recalde Tello; Angela Mishel Torres Cadena; Winna Viviana Arévalo Vaca

sencilla todo lo relacionado sobre la diabetes gestacional y sus implicaciones tanto en la mujer como en el desarrollo del feto.

\section{Resultados.}

Tabla 1. Interrogantes sobre la prescripción de la metformina

\begin{tabular}{|l|l|}
\hline \multicolumn{1}{|c|}{ Preguntas } & \multicolumn{1}{c|}{ Respuestas } \\
\hline ¿Cuándo se utiliza la metformina para el & La metformina es la biguanida más \\
manejo de la diabetes mellitus & $\begin{array}{l}\text { utilizada para el manejo de la diabetes } \\
\text { mellitus tipo 2, se está estudiando como } \\
\text { una alternativa útil y segura para el } \\
\text { manejo de la mujer con diabetes mellitus } \\
\text { gestacional; para disminuir los gastos y } \\
\text { mejorar el margen de seguridad que } \\
\text { implica el manejo con insulina. está } \\
\text { contraindicada en pacientes con deterioro } \\
\text { de la función renal, con deterioro de la } \\
\text { función hepática y en aquellas pacientes } \\
\text { que puedan cursar con hipoxia }\end{array}$ \\
\hline
\end{tabular}




\section{Manejo de la diabetes gestacional}

Vol. 3, núm. 3 Esp., (2019)

José Luis Maldonado López; Ivonne Carolina Recalde Tello; Angela Mishel Torres Cadena;

Winna Viviana Arévalo Vaca

\begin{tabular}{|l|l|}
\hline $\begin{array}{l}\text { ¿Cuáles son los criterios para iniciar con } \\
\text { el medicamento? }\end{array}$ & $\begin{array}{l}\text { Los criterios para iniciar el manejo } \\
\text { farmacológico son } 90 \mathrm{mg} / \mathrm{dL} \text { en ayuno y } \\
120 \mathrm{mg} / \mathrm{dL} \text { posprandial } 2 \text { horas }\end{array}$ \\
\hline $\begin{array}{l}\text { ¿Cuál es la dosis de metformina y cómo se } \\
\text { váxincrementando, cuál es la dosis }\end{array}$ & $\begin{array}{l}\text { Se debe de iniciar con 500-850 mg de } \\
\text { metformina con el alimento más } \\
\text { importante de la paciente e incrementos }\end{array}$ \\
$\begin{array}{l}\text { ¿Cuándo se debe agregar insulina al } \\
\text { manejo con metformina de la diabetes }\end{array}$ & $\begin{array}{l}\text { semanales de la dosis inicial, divididos en } \\
\text { a } 3 \text { tomas al día hasta que se alcance el } \\
\text { control metabólico. Estos incrementos se } \\
\text { deben de realizar por semana para } \\
\text { risminuir los riesgos de efectos }\end{array}$ \\
secundarios y hasta alcanzar la dosis \\
máxima descrita que es de 2 000 a 2 500 \\
mg por día.
\end{tabular}




\section{Manejo de la diabetes gestacional}

Vol. 3, núm. 3 Esp., (2019)

José Luis Maldonado López; Ivonne Carolina Recalde Tello; Angela Mishel Torres Cadena; Winna Viviana Arévalo Vaca

\begin{tabular}{|c|c|}
\hline & $\begin{array}{l}\text { máxima del medicamento, continúan con } \\
\text { descontrol de los niveles de glucemia en } \\
\text { ayuno o } 2 \text { horas postprandial }\end{array}$ \\
\hline $\begin{array}{l}\text { ¿Hay algún factor predictivo para conocer } \\
\text { que paciente con diabetes mellitus va a } \\
\text { necesitar usar insulina junto con la } \\
\text { metformina? }\end{array}$ & $\begin{array}{l}\text { El primer factor es cuando la edad } \\
\text { gestacional es menor en el momento del } \\
\text { diagnóstico de diabetes mellitus } \\
\text { gestacional, siendo mayor la probabilidad } \\
\text { de que se requiera el uso de la insulina. El } \\
\text { segundo factor son los niveles de glucosa } \\
\text { en ayuno al momento de realizar el } \\
\text { diagnóstico: mientras más elevado sean } \\
\text { los niveles de glucosa es mayor la } \\
\text { probabilidad de que se requiera insulina } \\
\text { para el manejo de la paciente. El tercer } \\
\text { factor son los niveles de fructosamina, que } \\
\text { se determinan al momento de realizar el } \\
\text { diagnóstico: los niveles más elevados de } \\
\text { fructosamina tienen un mayor riesgo de } \\
\text { utilizar insulina para el control de las } \\
\text { pacientes. }\end{array}$ \\
\hline
\end{tabular}




\section{Manejo de la diabetes gestacional}

Vol. 3, núm. 3 Esp., (2019)

José Luis Maldonado López; Ivonne Carolina Recalde Tello; Angela Mishel Torres Cadena;

Winna Viviana Arévalo Vaca

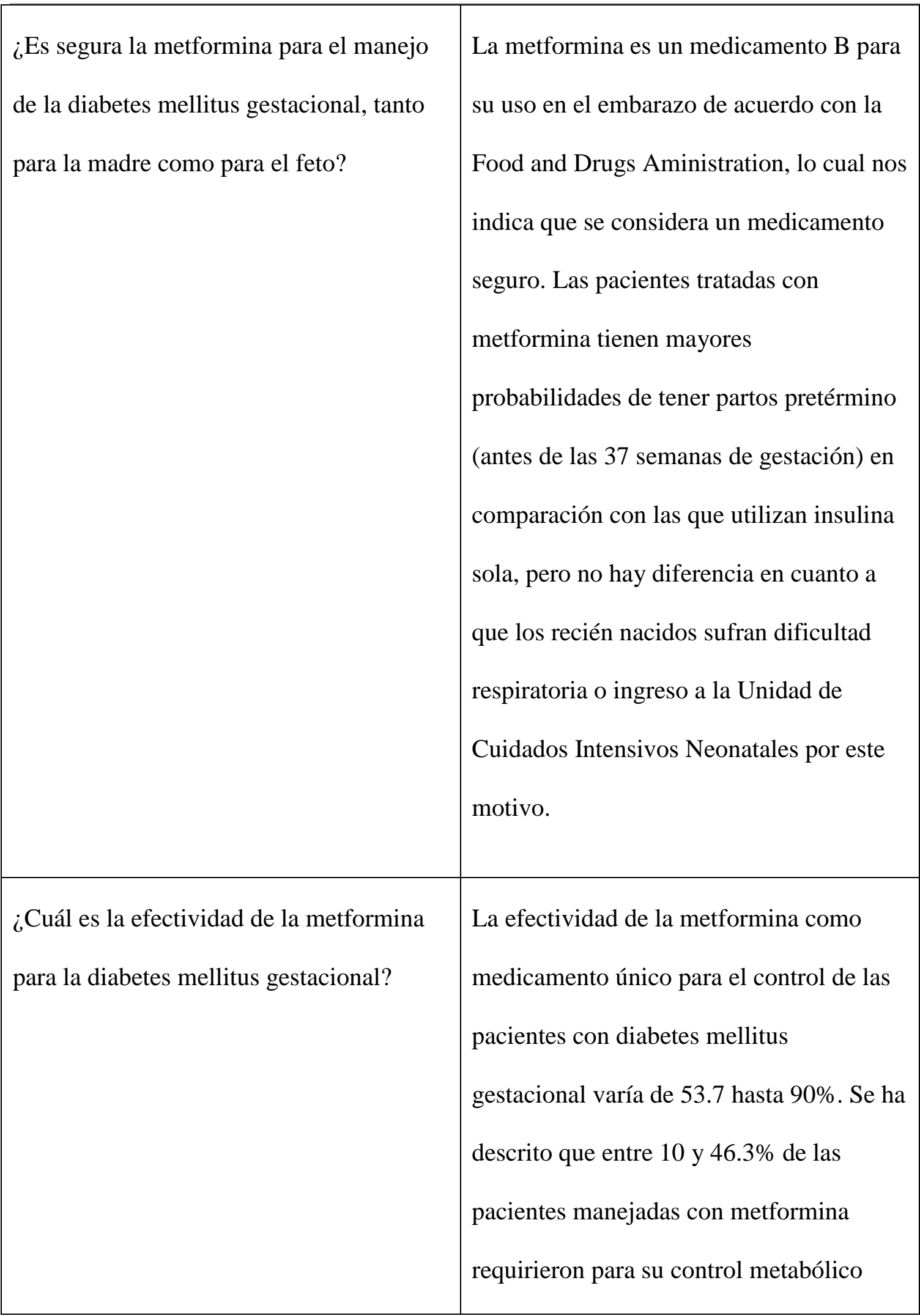




\section{Manejo de la diabetes gestacional}

Vol. 3, núm. 3 Esp., (2019)

José Luis Maldonado López; Ivonne Carolina Recalde Tello; Angela Mishel Torres Cadena; Winna Viviana Arévalo Vaca

\begin{tabular}{|l|l|}
\hline & insulina. \\
\hline ¿Cuáles fueron los principales efectos & Los efectos secundarios de la metformina \\
son predominantemente del tracto \\
digestivo: diarrea, flatulencia y molestias \\
intestinales; estos síntomas son \\
dependientes de la dosis y pueden \\
disminuir haciendo incrementos \\
paulatinos de la dosis \\
\hline
\end{tabular}

Fuente: (San Martín-Herrasti, Alcázar-Álvarez, Serrano-Berrones, Tirado-Chávez, \& CastroHerrera, 2014). Elaboración Propia.

Imagen 1. Criterios diagnósticos de diabetes mellitus gestacional

\begin{tabular}{|c|c|c|c|c|c|c|}
\hline \multirow{2}{*}{ Criterios diagnósticos } & \multirow{2}{*}{$\begin{array}{c}\text { Carga PTOG } \\
\text { (grs) }\end{array}$} & \multicolumn{4}{|c|}{ Puntos de corte de glicemia en el PTOG $(\geq)$ (mg/dl) } & \multirow{2}{*}{$\begin{array}{l}\text { Valores alterados } \\
\text { para diagnóstico }\end{array}$} \\
\hline & & Ayunas & 1-hora & 2-horas & 3-horas & \\
\hline O'Sullivan \& Mahan, 1964 & 100 & 90 & 165 & 145 & 125 & $\geqslant 2$ \\
\hline Carpenter \& Coustan, 1982 & 100 & 95 & 180 & 155 & 140 & $\geqslant 2$ \\
\hline OMS, 1999 & 75 & 126 & - & 140 & - & $\geqslant 1$ \\
\hline IADPSG, 2010 & 75 & 92 & 180 & 153 & - & $\geqslant 1$ \\
\hline
\end{tabular}

Fuente: (Trujillo, 2016).

Estos criterios descritos usan la prueba de tolerancia oral a la glucosa como prueba de diagnóstico, pudiendo ser la carga de 75 o 100 grs, y después de un ayuno de 10-14 horas. Sin embargo, esta prueba diagnóstica tiene algunas características desfavorables como no ser 


\section{Manejo de la diabetes gestacional}

Vol. 3, núm. 3 Esp., (2019)

José Luis Maldonado López; Ivonne Carolina Recalde Tello; Angela Mishel Torres Cadena; Winna Viviana Arévalo Vaca

fisiológica, desagradable al gusto, puede provocar náuseas y vómitos en las mujeres embarazadas, y además muestra una baja reproducibilidad. Las diferencias principales entre los criterios diagnósticos son: el número de muestras recolectadas durante la prueba de tolerancia oral a la glucosa (ayunas, 1-hora, 2-horas y/o 3-horas), los valores de los puntos de corte para glicemia plasmática, y el número de valores de glicemia plasmática alterados requeridos para el diagnóstico. No obstante, existen variantes de estos criterios, que son adaptaciones de acuerdo a los estándares de salud de cada país o región. Estas diferencias entre el criterio diagnóstico y el tamizaje empleado en cada región han resultado en la variación de la prevalencia/incidencia para cada región geográfica, junto con las características étnicas y sociales de la población en cuestión (Trujillo, 2016, pág. 1252).

De manera general se va aceptando que la diabetes gestacional no es una forma diferente de diabetes sino una etapa de la misma enfermedad que ocurre en personas predispuestas genéticamente en la que se suma el impacto del estilo de vida. El aumento en la prevalencia de obesidad y el retardo en la edad de inicio de la etapa reproductiva de la mujer sustentan la teoría de un aumento en la ocurrencia de la diabetes gestacional (Zárate, Saucedo, Basurto, \& Hernández, 2011, pág. 2).

El tratamiento farmacológico se debe considerar cuando la dieta y el ejercicio no logran las cifras meta (menor de $95 \mathrm{mg} / \mathrm{dL}$ en ayuno y $140 \mathrm{mg} / \mathrm{dL}$ una hora posprandial) para el control de la glucosa en sangre en un periodo de dos semanas.1, 28 (E-III) (Algoritmo 2). Los análogos de insulina lispro y aspart, así como la insulina humana de acción rápida y la insulina de acción intermedia han sido investigadas en el embarazo y se ha demostrado su efectividad y seguridad clínica, mínimo transporte a través de la placenta y no se ha informado teratogenicidad (E-I).2 


\section{Manejo de la diabetes gestacional}

Vol. 3, núm. 3 Esp., (2019)

José Luis Maldonado López; Ivonne Carolina Recalde Tello; Angela Mishel Torres Cadena; Winna Viviana Arévalo Vaca

Además, se han identificado menos episodios de hipoglucemia, un mejor control de la glucemia posprandial y mayor satisfacción de la paciente. La insulina de acción intermedia se utiliza en combinación con las anteriores.29 (E-Ib) (Font-López, y otros, 2010, pág. 677).

La piedra angular del tratamiento de DMG sigue siendo la asesoría nutricional y la intervención dietética. La dieta óptima debe satisfacer las necesidades calóricas y nutricionales para sustentar el embarazo, sin dar como resultado una hiperglucemia postprandial significativa. En la mayoría de los casos, las mujeres con DMG pueden recibir indicaciones sobre la dieta y enseñanza del auto manejo de la glucosa en la sangre, en un ambiente de consulta externa. Una vez establecido el diagnóstico, las mujeres comienzan un programa dietético de 2,000 a 2,500 kcal diarias. Esto representa aproximadamente $35 \mathrm{kcal} / \mathrm{kg}$ del peso del embarazo en curso. Una dieta compuesta de 50 a $60 \%$ de carbohidratos con frecuencia daría como resultado un aumento excesivo de peso e hiperglucemia postprandial. Por esta razón, se ha sugerido que la ingestión de carbohidratos se limite a 33-40\% de las calorías.Los carbo hidratos complejos son preferibles a los carbohidratos simples porque es menos probable que produzcan hiperglucemia postprandial significativa. Sin embargo, los estudios aleatorios con potencia estadística adecuada carecen de pruebas para determinar el beneficio de las dietas de carbohidratos de bajo índice glucémico en la DMG. En la práctica se recomiendan tres comidas y dos o tres refrigerios para distribuir la ingestión de glucosa y minimizar las acometidas de glucosa postprandial (Landon \& Gabbe, 2011, pág. 11).

La dieta, en particular, reduce significativamente el riesgo de pre-eclampsia, diabetes gestacional, hipertensión gestacional, y los nacimientos prematuros en comparación con cualquier otra intervención. Las intervenciones basadas en una dieta balanceada de $18-24 \mathrm{~kJ} / \mathrm{kg}$, 


\section{Manejo de la diabetes gestacional}

Vol. 3, núm. 3 Esp., (2019)

José Luis Maldonado López; Ivonne Carolina Recalde Tello; Angela Mishel Torres Cadena;

Winna Viviana Arévalo Vaca

una dieta con bajos niveles de glucosa, que incluya granos enteros sin procesar, frutas, legumbres y verduras se han descrito como más beneficiosas. Una dieta que incluya un 30\% de grasa, 15 a $20 \%$ de proteína, y 50 a $55 \%$ de hidratos de carbono, con la ingesta de energía individualizada a las necesidades de la madre debería de ser adecuada para una ganancia de peso correcta durante el embarazo (Bustillo, y otros, 2016, pág. 4).

En la DG se debe corregir la hiperglucemia que rebase los límites considerados como normales en el embarazo mediante una terapéutica individualizada. Para este propósito tanto la alimentación adecuada y la actividad física regular son insoslayables, pero en caso de ser insuficientes se agregan los fármacos como son la insulina humana sintética, las sulfonilureas (exclusivamente por el momento gliburida) y recientemente la metformina. En casos de mínima hiperglucemia postprandial también se ha utilizado acarbosa para reducir la absorción intestinal de glucosa. La selección del fármaco depende de la aceptabilidad, costo y efectividad, tomando en cuenta que generalmente hay preferencia por la medicación oral, aunque lo más efectivo sigue siendo la insulina. Ninguno de estos recursos terapéuticos es teratogénico y pueden utilizarse en forma combinada (Zárate, Hernández-Valencia, \& Saucedo, La detección y manejo de la diabetes gestacional. Controversias, críticas y comentarios, 2008).

Tabla 2. Manejo de la diabetes gestacional

\begin{tabular}{|l|l|} 
Plan de Nutrición & $\begin{array}{l}\text { El plan de nutrición incluye: 1) evaluación basada en historia } \\
\text { clínica, exámenes de laboratorio, hábitos, preferencias y recursos } \\
\text { destinados a la alimentación; 2) estrategias para controlar el }\end{array}$ \\
\hline
\end{tabular}




\section{Manejo de la diabetes gestacional}

Vol. 3, núm. 3 Esp., (2019)

José Luis Maldonado López; Ivonne Carolina Recalde Tello; Angela Mishel Torres Cadena; Winna Viviana Arévalo Vaca

\begin{tabular}{|c|c|}
\hline & $\begin{array}{l}\text { consumo de hidratos de carbono para promover hábitos de } \\
\text { nutrición que permitan alcanzar las metas de glucemia, aumentar } \\
\text { de peso en forma razonable y evitar la aparición de cetonurias; 3) } \\
\text { programar consultas subsecuentes para vigilar el aumento de peso, } \\
\text { las cifras de glucemia y la capacidad de la paciente para llevar el } \\
\text { plan de alimentación }\end{array}$ \\
\hline Automonitoreo & $\begin{array}{l}\text { El automonitoreo de glucosa en sangre permite que el equipo } \\
\text { médico realice una valoración objetiva del control de la glucemia } \\
\text { en forma cotidiana, y conozca el efecto de las comidas y } \\
\text { colaciones sobre la glucemia. También ayuda a establecer si es } \\
\text { necesario modificar las proporciones de macronutrimentos } \\
\text { recomendadas. En condiciones ideales, el automonitoreo debe } \\
\text { realizarse cinco veces al día: en ayunas, antes del desayuno, de la } \\
\text { comida, de la cena y antes de acostarse. La frecuencia del } \\
\text { automonitoreo puede reducirse a tres o cuatro veces al día si se } \\
\text { llega a establecer un contol adecuado de glucosa }\end{array}$ \\
\hline Aplicación de & $\begin{array}{l}\text { Hasta la fecha, la insulina es el único recurso farmacológico } \\
\text { autorizado en el control de la glucemia durante el embarazo. El uso } \\
\text { de insulina se recomienda cuando el plan de nutrición no logra } \\
\text { alcanzar o mantener las metas de glucemia por automonitoreo }\end{array}$ \\
\hline
\end{tabular}




\section{Manejo de la diabetes gestacional}

Vol. 3, núm. 3 Esp., (2019)

José Luis Maldonado López; Ivonne Carolina Recalde Tello; Angela Mishel Torres Cadena;

Winna Viviana Arévalo Vaca

\begin{tabular}{|c|l|}
\hline $\begin{array}{c}\text { Antidiabéticos } \\
\text { orales }\end{array}$ & $\begin{array}{l}\text { Hasta la fecha, no está autorizado el uso de antidiabéticos orales en } \\
\text { el manejo de la diabetes gestacional, aunque se han valorado en } \\
\text { este campo desde hace muchos años. Los principales argumentos } \\
\text { que han impedido su uso en este grupo de pacientes han sido la } \\
\text { elevada morbilidad y mortalidad fetal en las mujeres que los } \\
\text { consumían antes del embarazo y los siguieron utilizando durante la } \\
\text { gestación, y el riesgo de efectos fetales por el paso de estos } \\
\text { fármacos a través de la membrana placentaria. }\end{array}$ \\
\hline
\end{tabular}

Fuente: (Duarte-Gardea, Muñoz, Rodríguez-Saldaña, \& Escorza-Domínguez, 2004).

\section{Conclusiones.}

En términos generales la diabetes gestacional es una condición muy común y riesgosa en el embarazo, es más frecuente en mujeres que presentan una condición de obesidad, sin embargo, con los tratamientos adecuados y siguiéndose lo planes sugeridos por los médicos es posible llevar a término de manera exitosa y sin riesgo tanto para la madre como para el feto el embarazo.

La metformina es el tratamiento más empleado y sugerido cuando se presentan procesos de diabetes gestacional mucho más que la insulina, ya que es menos invasiva y en comparaciones con otros tratamientos tiene mucho éxito para el término del embarazo. 


\section{Manejo de la diabetes gestacional}

Vol. 3, núm. 3 Esp., (2019)

José Luis Maldonado López; Ivonne Carolina Recalde Tello; Angela Mishel Torres Cadena; Winna Viviana Arévalo Vaca

Los estilos de vida saludables son determinantes para reducir los riesgos de padecer diabetes gestacional en el embarazo, así como una buena dieta.

\section{Bibliografía.}

Arizmendi, J., Pertuz, V., Colmenares, A., Hoyos, D., \& Palomo, T. (2012 de noviembre de 2012). Diabetes gestacional y complicaciones neonatales. Revista Med de la Facultad de Medicina, 20(2), 50-60.

Bustillo, A. L., Melendez, W., Urbina, L., Nuñez, J., Eguigurems, D., Pineda, C., \& Bustillo, L. (2016). Sobrepeso y obesidad en el embarazo: complicaciones y manejo. Archivos de medicina, 12(3), 1-7.

Duarte-Gardea, M., Muñoz, G., Rodríguez-Saldaña, J., \& Escorza-Domínguez, A. (2004). Prevalencia, detección y tratamiento de la diabetes gestacional. Revista Salud Pública y Nutrición, 5(1), 1-9.

Font-López, K. C., Cejudo-Carranza, E., López-Caucana, A., Peralta-Pedrero, M., DíazVelásquez, M., Puello-Tamara, E., \& Ramírez-Torres, A. (2010). Guía de práctica clínica. Diagnóstico y tratamiento de la diabetes en el embarazo. Revista Médica del Instituto Mexicano del Seguro Social, 48(6), 673-684.

Landon, M., \& Gabbe, S. (diciembre de 2011). Gestational diabetes mellitus. Obstetrics \& Gynecology, 118(6), 1379-93.

Rimbao Torres, G., Cruz Hernández, J., Safora Enríquez, O., Rodríguez Izquierdo, A., Morales Chamizo, M., \& Velasco Boza, A. (2007). Comportamiento de la diabetes gestacional en el embarazo en la adolescencia. Revista Cubana de Medicina General Integral, 23(3), 1561-3038.

San Martín-Herrasti, J. M., Alcázar-Álvarez, L., Serrano-Berrones, M., Tirado-Chávez, J., \& Castro-Herrera, G. (2014). Metformina y diabetes gestacional. Revista de Especialidades Médico-Quirúrgicas, 19(3), 347-355.

Trujillo, J. (2016). Critérios diagnósticos y efectividad de intervenciones para el manejo de diabetes gestacional. Revista Cuidarte, 7(2), 1251-1254.

Zárate, A., Hernández-Valencia, M., \& Saucedo, R. (2008). La detección y manejo de la diabetes gestacional. Controversias, críticas y comentarios. Acta Méd Grupo Ángeles, 6(3), 130-2.

Zárate, A., Saucedo, R., Basurto, L., \& Hernández, M. (2011). El nuevo enfoque hacia la diabetes gestacional. Revista Médica del Instituto Mexicano del Seguro Social, 49(1), 13. 


\section{Manejo de la diabetes gestacional}

Vol. 3, núm. 3 Esp., (2019)

José Luis Maldonado López; Ivonne Carolina Recalde Tello; Angela Mishel Torres Cadena;

Winna Viviana Arévalo Vaca

$$
\text { (9) (1) }(2)
$$

RECONOCIMIENTO-NOCOMERCIAL-COMPARTIRIGUAL

CC BY-NC-SA

ESTA LICENCIA PERMITE A OTROS ENTREMEZCLAR, AJUSTAR Y CONSTRUIR A PARTIR DE SU OBRA CON FINES NO

COMERCIALES, SIEMPRE Y CUANDO LE RECONOZCAN LA AUTORÍA Y SUS NUEVAS CREACIONES ESTÉN BAJO UNA LICENCIA CON LOS MISMOS TÉRMINOS. 